\title{
An asymmetric dihydroxylation route towards the synthesis of $(R)$-clorprenaline hydrochloride
}

\author{
Rajesh Kumar Pandey ${ }^{* \dagger}$, Rajesh Kumar Upadhyay, and Pradeep Kumar \\ Division of Organic Chemistry: Technology, National Chemical Laboratory, Pune-411008, India \\ E-mail: rkpandey1@yahoo.com
}

\begin{abstract}
An efficient asymmetric synthesis of enantiomerically pure $(R)$-clorprenaline hydrochloride is described using Sharpless asymmetric dihydroxylation as the key step.
\end{abstract}

Keywords: $(R)$-Clorprenaline hydrochloride, asymmetric dihydroxylation, enantioselective synthesis

\section{Introduction}

Due to the high demand and preference for the use of enantiomerically pure drugs, there has been an upsurge of interest in the development of potentially useful processes for the asymmetric synthesis of pharmaceutical products. (R)-Clorprenaline hydrochloride $\mathbf{1}$ (Fig. 1) is a $\beta_{2}$-agonist that is effective for the treatment of diverse disease states such as bronchitis and asthma ${ }^{1}$ with only few reports of its synthesis in the literature. ${ }^{2}$ As a part of our research program aimed at developing enantioselective synthesis of naturally occurring lactones and amino alcohols, ${ }^{3}$ the Sharpless asymmetric dihydroxylation ${ }^{4}$ was envisaged as a powerful tool to obtain chiral dihydroxy compounds offering considerable opportunities for synthetic manipulation. Herein we report a new and highly enantioselective synthesis of $(R)$-clorprenaline hydrochloride by employing Sharpless asymmetric dihydroxylation of $o$-chlorostyrene $\mathbf{2}$ as the key step.

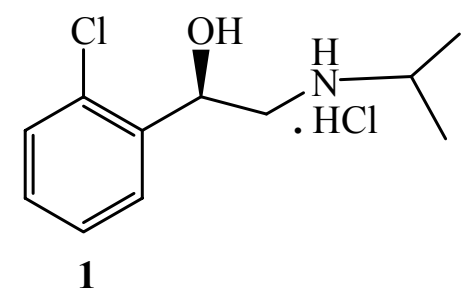

\section{Figure 1}

\footnotetext{
${ }^{\dagger}$ Current Address: Department of Chemistry, Marquette University, Milwaukee, WI-53233, USA. Fax: +001 414 2887066
} 


\section{Results and Discussion}

The synthesis of $(R)$-clorprenaline hydrochloride $\mathbf{1}$ started from $o$-chlorostyrene $\mathbf{2}$, a commercially available starting material is shown in Scheme 1. The dihydroxylation of $o$ chlorostyrene $\mathbf{2}$ with osmium tetroxide and potassium ferricyanide as co-oxidant under Sharpless asymmetric dihydroxylation ${ }^{4}$ gave the diol 3 in $94 \%$ yield with $95 \%$ ee, ${ }^{5}[\alpha]_{\mathrm{D}}{ }^{25}=$ 72.22 (c 2, EtOH). Selective conversion of the primary hydroxyl group of $\mathbf{3}$ into a tosylate with tosyl chloride in the presence of catalytic amount of dibutyltin oxide ${ }^{6,3 e}$ afforded 4 in excellent yield. We failed in our attempt to synthesise $\mathbf{1}$ from $\mathbf{4}$ by nucleophilic displacement with isopropylamine. Accordingly the diol $\mathbf{3}$ was converted into cyclic sulfite which on reaction with isopropylamine gave only the starting material back. In an alternative approach, the tosylate 4 was converted into the epoxide 5 , ${ }^{2}$ which on regioselective opening with isopropyl amine followed by treatment with $0.5 \mathrm{M} \mathrm{HCl}$ in methanol gave the target compound 1 as hydrochloride in excellent yield. The physical and spectroscopic data of $\mathbf{1}$ were in full agreement with the literature values. ${ }^{2}$
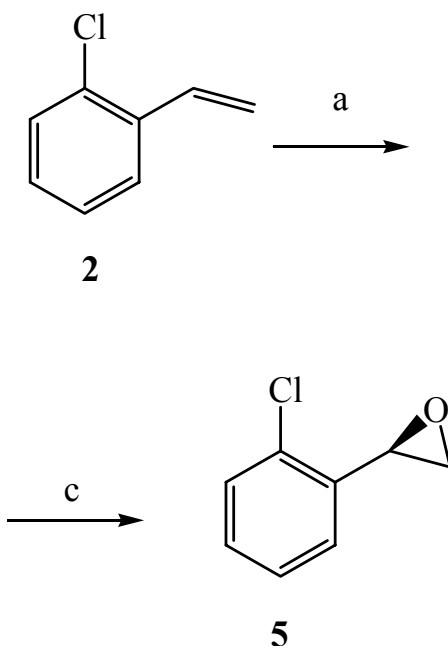

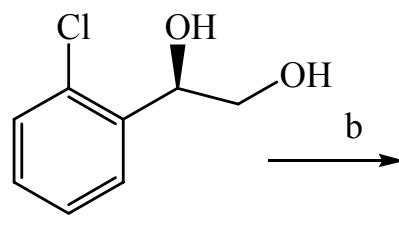

3

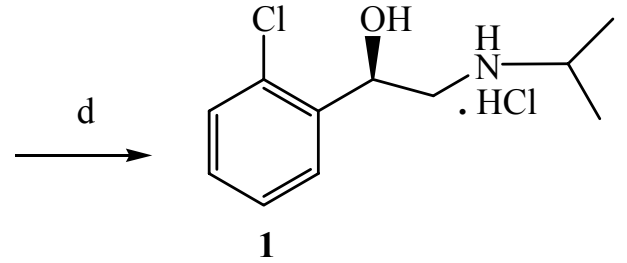

Scheme 1. Reagents and conditions: (a) (DHQD) ${ }_{2} \mathrm{PHAL}, \mathrm{K}_{3} \mathrm{Fe}(\mathrm{CN})_{6}, \mathrm{~K}_{2} \mathrm{CO}_{3}, \mathrm{t}-\mathrm{BuOH}: \mathrm{H}_{2} \mathrm{O}$, $\mathrm{OsO}_{4}, 0^{\circ} \mathrm{C}, 24 \mathrm{~h}, 94 \%$. (b) dibutyltin oxide (0.2 mol\%), TsCl, $\mathrm{NEt}_{3}, \mathrm{CH}_{2} \mathrm{Cl}_{2}, \mathrm{rt}, 45 \mathrm{~min}, 95 \%$. (c) $\mathrm{NaOH} / \mathrm{MeOH}, 6$ h, 95\%; (d) isopropylamine, $\mathrm{HCl} / \mathrm{MeOH}, 98 \%$.

In summary, a practical and highly enantioselective synthesis of $(R)$-clorprenaline $\mathbf{1}$, has been achieved using the Sharpless asymmetric dihydroxylation as the key step and source of chirality. The synthetic strategy described can be further extended to other enantiomers and related analogs. 


\section{Experimental Section}

General Procedures. The solvents were purified and dried by standard procedure prior to use; petroleum ether of boiling range $60-80^{\circ} \mathrm{C}$ was used. Optical rotation was measured using sodium D line on a JASCO-P-1020-polarimeter. Infrared spectra were recorded on Perkin Elmer FT-IR spectrometer. The Mass spectra were recorded either with GC MS or with a Finnigan LCMS mass spectrometer. Enantiomeric excess was measured using either the chiral HPLC or by comparison with optical rotation.

(R)-(-)-o-Chlorophenylethanediol (3). To a mixture of $\mathrm{K}_{3} \mathrm{Fe}(\mathrm{CN})_{6}(10.67 \mathrm{~g}, 32.49 \mathrm{mmol})$, $\mathrm{K}_{2} \mathrm{CO}_{3}(4.49 \mathrm{~g}, 32.49 \mathrm{mmol})$ and (DHQD) ${ }_{2} \mathrm{PHAL}(0.085 \mathrm{~g}, 0.011 \mathrm{mmol})$ in $t-\mathrm{BuOH}-\mathrm{H}_{2} \mathrm{O}(1: 1,55$ $\mathrm{mL})$ cooled to $0^{\circ} \mathrm{C}$ was added osmium tetroxide $(0.44 \mathrm{ml}, 0.1 \mathrm{M}$ solution in toluene). After stirring for $5 \mathrm{~min}$ at $0^{\circ} \mathrm{C}$, the olefin $2(1.5 \mathrm{~g}, 10.83 \mathrm{mmol})$ was added in one portion. The reaction mixture was stirred at $0^{\circ} \mathrm{C}$ for $24 \mathrm{~h}$ and then quenched with solid sodium sulfate $(2 \mathrm{~g})$, the stirring was continued for an additional $45 \mathrm{~min}$ and then solution was extracted with EtOAc $(3 \times 50 \mathrm{~mL})$. The combined organic phase were dried $\left(\mathrm{Na}_{2} \mathrm{SO}_{4}\right)$ and concentrated. Silica gel column chromatography of the crude product using petroleum ether: EtOAc (1:1) as eluent gave diol 3 $(1.76 \mathrm{~g}, 94 \%)$ as a white solid. mp $98-99^{\circ} \mathrm{C} ;[\alpha]_{\mathrm{D}}{ }^{25}=-72.22(c$ 2, EtOH $)\left[\right.$ Lit. $^{2 \mathrm{a}}[\alpha]_{\mathrm{D}}{ }^{22}-57^{\circ}(\mathrm{c}$ $1.8, \mathrm{EtOH})$ for $85 \%$ ee]. ${ }^{1} \mathrm{H}$ NMR $\left(200 \mathrm{MHz}, \mathrm{CDCl}_{3}\right): \delta 3.21(\mathrm{bs}, 2 \mathrm{H}), 3.30(\mathrm{~d}, J=2 \mathrm{~Hz}, 1 \mathrm{H})$, $3.63(\mathrm{dd}, J=4.3,2 \mathrm{~Hz}, 1 \mathrm{H}), 4.98$ (dd, $J=4.5,2.3 \mathrm{~Hz}, 1 \mathrm{H}), 7.12-7.4$ (m, 4H). IR $\left(\mathrm{CHCl}_{3}\right): 3401$, 2328, 2818, 1604, 1590, $\mathrm{cm}^{-1}$; Mass (ESI): $172\left(\mathrm{M}^{+}\right)$. Anal. Calcd for $\mathrm{C}_{8} \mathrm{H}_{9} \mathrm{ClO}_{2}: \mathrm{C}, 55.67 ; \mathrm{H}$, 5.26; Cl, 20.54. Found: C, 55.63; H, 5.30; Cl, 20.50.

(R)-(-)-Toluene-4-sulfonic acid 2-hydroxy-2-o-chlorophenylethyl ester (4). To a mixture of diol $3(1.0 \mathrm{~g}, 5.76 \mathrm{mmol})$ in dry dichloromethane $(12 \mathrm{ml})$ was added dibutyltin oxide $(0.003 \mathrm{~g}$, $0.2 \mathrm{~mol} \%$ of diol ) followed by the addition of $p$-toluenesulfonyl chloride $(1.10 \mathrm{~g}, 5.76 \mathrm{mmol})$ and triethylamine $(0.586 \mathrm{~g}, 5.76 \mathrm{mmol})$ and reaction was stirred at room temperature under nitrogen. The reaction was monitored by TLC. After completion of reaction (45 min), the mixture was quenched by adding water. The solution was extracted with dichloromethane $(3 \mathrm{x}$ $15 \mathrm{~mL})$ and then combined organic phase was washed with water, dried $\left(\mathrm{Na}_{2} \mathrm{SO}_{4}\right)$ and concentrated. Silica gel column chromatography of crude product using petroleum ether: EtOAc (7:3) as eluent afforded the monotosyl compound $4(1.80 \mathrm{~g}, 95 \%)$ as a viscous liquid. $[\alpha]_{\mathrm{D}}{ }^{25}=-$ $52.53\left(c 1.02, \mathrm{CHCl}_{3}\right) ;{ }^{1} \mathrm{H}$ NMR $\left(200 \mathrm{MHz}, \mathrm{CDCl}_{3}\right): \delta 1.70(\mathrm{~s}, 1 \mathrm{H}, \mathrm{OH}), 2.10(\mathrm{~s}, 3 \mathrm{H}), 3.68(\mathrm{~m}$, 1H), $3.96(\mathrm{~m}, 1 \mathrm{H}), 5.05$ (dd, $1 \mathrm{H}, J=8.8,2.9 \mathrm{~Hz}), 6.96(\mathrm{~m}, 4 \mathrm{H}), 7.29-7.50$ (m, 4H). IR (Neat): 3525, 3021, 2951, 1598, $1215 \mathrm{~cm}^{-1}$. Mass (ESI): $326\left(\mathrm{M}^{+}\right)$. Anal. Calcd for $\mathrm{C}_{15} \mathrm{H}_{15} \mathrm{ClO}_{4} \mathrm{~S}: \mathrm{C}$, 55.13; H, 4.63; Cl, 10.85; S, 9.81 Found: C, 55.18; H, 4.50, Cl, 10.70; S, 9.90.

(R)-1-(2-Chlorophenyl)-1,2-epoxypropane (5). ${ }^{\text {2a }}$ The tosyl compound 4 (1.6 g, $\left.4.89 \mathrm{mmol}\right)$ was dissolved in methanol $(14 \mathrm{~mL})$. The mixture was cooled in ice-salt bath before the methanolic solution $(14 \mathrm{~mL})$ of $\mathrm{NaOH}(0.22 \mathrm{~g}, 5.6 \mathrm{mmol})$ was added. The reaction mixture was stirred at $10^{\circ} \mathrm{C}$ for $6 \mathrm{~h}$. After that it was diluted with $100 \mathrm{ml}$ ether and water $(25 \mathrm{~mL})$ was added. The organic layer was separated and washed with water, dried. Removal of the solvent and purification of residue by silica gel column chromatography using pet ether: ethyl acetate (50:1) 
as eluent furnished the epoxide $5(722 \mathrm{mg}, 95 \%)$ as colorless oil. $[\alpha]_{\mathrm{D}}{ }^{25}=-56.93\left(\mathrm{c} 1, \mathrm{CHCl}_{3}\right)$ $\left[\right.$ Lit. $^{2 \mathrm{a}}[\alpha]_{\mathrm{D}}{ }^{22}-43.4^{\circ}$ (c 1.5, $\mathrm{CHCl}_{3}$ for $65 \%$ ee]. ${ }^{1} \mathrm{H}$ NMR $\left(200 \mathrm{MHz}, \mathrm{CDCl}_{3}\right): 2.69(\mathrm{dd}, J=5.8$, $2.4 \mathrm{~Hz}, 1 \mathrm{H},), 3.22$ (dd, $J=5.8,4.1 \mathrm{~Hz}, 1 \mathrm{H}), 4.23$ (dd, $J=4.3,2.4 \mathrm{~Hz}, 1 \mathrm{H}$,$) , 7.26-7.39 (m, 4H); MS$ $\mathrm{m} / \mathrm{z}: 154\left(\mathrm{M}^{+}\right)$.

(R)-Clorprenaline hydrochoride (1). A solution of epoxide 5 (0.2 g, $1.29 \mathrm{mmol})$, excess of isopropyl amine $(4 \mathrm{~mL})$ and 4 drops of water was stirred at room temperature for $30 \mathrm{~min}$. After that mixture was heated at $60^{\circ} \mathrm{C}$ for $6 \mathrm{~h}$, after removal of the excess isopropyl amine, the residue was dissolved in $50 \mathrm{~mL}$ ether, filtered, dried and concentrated. The solid was recrystallized from hexane to afford $(R)$-clorprenaline $(270 \mathrm{mg}, 98 \%)$ as a white solid, mp $79-80^{\circ} \mathrm{C} ;[\alpha]_{\mathrm{D}}{ }^{25}=-69.21$

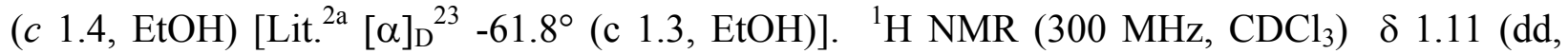
$J=6.2,4.2 \mathrm{~Hz}, 6 \mathrm{H}), 2.47(\mathrm{dd}, J=12.2,9 \mathrm{~Hz}, 1 \mathrm{H}), 2.70-2.90(\mathrm{~m}, 3 \mathrm{H}), 3.00(\mathrm{dd}, J=12.2,3.3 \mathrm{~Hz}$, $1 \mathrm{H}), 5.02(\mathrm{dd}, J=8.9,3.3 \mathrm{~Hz}, 1 \mathrm{H}), 7.18-7.50(\mathrm{~m}, 4 \mathrm{H}): \quad \mathrm{IR}\left(\mathrm{CHCl}_{3}\right): 3320,3018,2982,2788$, $2704,1593,763 \mathrm{~cm}^{-1}$

The hydrochloride was obtained as follow: The clorprenaline was dissolved in methanol ( $1 \mathrm{ml})$, and $2 \mathrm{ml}$ of $0.5 \mathrm{M} \mathrm{HCl}$ methanol was added. Removal of the solvent afforded $(R)$-clorprenaline hydrochloride as a white solid. The physical and spectroscopic data were in full agreement with the literature. ${ }^{2}$

\section{Acknowledgements}

RKP thanks CSIR, New Delhi for financial assistance.

\section{Reference and Footnotes}

1. Tatsumi, K.; Arima, N.; Yamato, C.; Yoshimura, H.; Tsukamoto, H. Chem. Pharm. Bull. 1970, 18.1254.

2. (a) Wei, Z-L; Li, Z-Y; Lin, G-Q, Tetrahedron 1998, 54, 13059. (b) Noe, C. R.; Knollmueller, M.; Gaertner, P.; Fleischhacker, W.; Katikarides, E. Monatshefte fuer Chemie 1995, 126, 557. (c) Ou, Z-M; Jin, Z-H; Wu, J-P; Yang, L-R; Cen, P-L, Gaoxiao Huaxue Gongcheng Xuebao 2005, 19, 511. (d) Furuhashi, K.; Takagi, K. Jpn. Kokai Tokkyo Koho 1986, 6 .

3. (a) Pandey R. K.; Fernandes, R. A.; Kumar, P. Tetrahedron Lett. 2002, 43, 4425. (b) Naidu, S. V.; Kumar, P. Tetrahedron Lett. 2003, 44, 1035. (c) Kandula, S. V.; Kumar, P. Tetrahedron Lett. 2003, 44, 1957. (d) Pandey, R. K; Upadhyay, P. K.; Kumar, P. Tetrahedron Lett. 2003, 44, 6245. (e) Kumar, P.; Upadhyay, R. K.; Pandey, R. K. Tetrahedron Asymmetry 2004, 15, 3955. (f) Pandey, S. K.; Kandula, S. V.; Kumar, P. Tetrahedron Lett. 2004, 45, 5877. (g) Pandey, S. K.; Kumar, P. Tetrahedron Lett. 2005, 46, 
4091. (h) Kumar, P.; Bodas, M. S. J. Org. Chem. 2005, 70, 360. (i) Kumar, P.; Naidu, S. V. J. Org. Chem. 2005, 70, 4207. (j) Kumar, P.; Naidu, S. V.; Gupta, P. J. Org. Chem. 2005, 70, 2843-2846. (k) Sadyandy, R.; Fernandes, R. A.; Kumar, P. Arkivoc 2005, 3, 36.

4. (a) Kolb, H. C.; VanNieuwenhze, M. S.; Sharpless, K. B. Chem. Rev. 1994, 94, 2483. (b) Becker, H.; Sharpless, K. B. Angew. Chem., Int. Ed. 1996, 35, 448.

5. The enantiomeric excess was determined by HPLC. HPLC model: Merck-Hitachi Lachrom Photo Diode Array detector (PDA); Column: Astec Cyclobond I (4.6 mm ID x $250 \mathrm{mmL}$ ); Mobile phase: methanol: water: (40: 60); Flow: $1 \mathrm{ml} / \mathrm{min}$.

6. Martinelli, M. J.; Vaidyanathan, R.; Pawlak, J. M.; Nayyar, N. K.; Dhokte, U. P.; Doecke, C. W.; Zollars, L. M. H.; Moher, E. D.; Khau, V. V.; Kosmrlj, B. J. Am. Chem. Soc. 2002, 124, 3578 